\title{
Stabilisation of medically refractory ventricular arrhythmia by intra-aortic balloon counterpulsation
}

G D Fotopoulos, M J Mason, S Walker, N S Jepson, D J Patel, A G Mitchell, C D J Ilsley, V E Paul

\begin{abstract}
Objective-To review the efficacy of intraaortic balloon counterpulsation (IABCP) in medically refractory ventricular arrhythmia.

Design-Retrospective analysis of the outcome of patients with ventricular arrhythmia treated with IABCP after transfer between 1992 and 1997.

Setting-Tertiary cardiac referral centre. Patients-21 patients (mean age 58 years) who underwent IABCP for control of ventricular arrhythmia. All had significant left ventricular impairment (mean ejection fraction $28.6 \%$ ); 18 had coronary artery disease.
\end{abstract}

Results-Before IABCP, 10 patients had incessant monomorphic ventricular tachycardia and 11 had paroxysmal ventricular tachycardia and/or ventricular fibrillation (VT/VF). IABCP resulted in suppression of ventricular arrhythmia in 18 patients, of whom 13 were weaned from IABCP. After stabilisation of ventricular arrhythmia, 10 patients were maintained on medical treatment alone and one underwent endocardial resection. IABCP was maintained until cardiac transplantation in five patients. One patient had a fatal arrest before discharge and one died from progressive heart failure. IABCP failed to control ventricular arrhythmia in three patients and was subsequently discontinued. A cardiac assist device was employed in one of these until cardiac transplantation; the other two were eventually stabilised on medical treatment. Nineteen patients were discharged from hospital. Overall survival was $95 \%$ at mean follow up of $\mathbf{2 5 . 7}$ months.

Conclusions-IABCP can be an effective means of controlling refractory ventricular arrhythmia, allowing time for the institution of more definitive treatment. (Heart 1999;82:96-100)

Keywords: ventricular arrhythmia; intra-aortic balloon counterpulsation

In patients with impaired left ventricular function, medically refractory ventricular arrhythmia can present a difficult management problem. Although an underlying arrhythmogenic substrate is necessary, the arrhythmia itself will compromise an already impaired haemodynamic state, by increasing myocardial ischaemia or left ventricular wall distension, and so exacerbate the situation. This can lead to a vicious circle of a worsening haemodynamic condition in conjunction with an increasingly arrhythmogenic myocardium. In such circumstances non-pharmacological methods of arrhythmia management such as endocardial resection and ventricular tachycardia ablation are currently employed, despite the high risk of these procedures in the unstable patient.

An alternative physical method that suppresses the arrhythmia and stabilises the haemodynamic status may allow time for implementation of appropriate drug treatment or enhance the patient's condition before a non-pharmacological approach.

Intra-aortic balloon counterpulsation (IABCP) improves coronary flow and reduces myocardial distension, thus potentially influencing ventricular irritability by direct and indirect effects. Previous reports ${ }^{1-3}$ have shown the efficacy of IABCP in the control of ventricular arrhythmias after myocardial infarction, yet the arrhythmia itself is rarely considered a primary indication for IABCP use.

In this report we describe a retrospective review of the efficacy of IABCP in controlling ventricular arrhythmia in patients resistant to antiarrhythmic drug treatment, repeated dc cardioversion, and pacing strategies.

\section{Methods}

Between 1992 and 1997, 21 patients (17 male, four female) were transferred to our unit (a cardiac tertiary referral centre) with medically resistant ventricular arrhythmia for further management, and were considered suitable for IABCP. The mean age for the group was 58 years (range 44 to 72 years). Consideration of IABCP was given to any patient with ventricular arrhythmia refractory to conventional treatment, but who would be suitable for more definitive treatment or who might otherwise have a reasonable quality of life if the arrhythmia were controlled.

Clinical details are shown in table 1. Eighteen patients had ischaemic heart disease. Five had suffered acute myocardial infarction between three and 15 days before the onset of the arrhythmia, 12 had evidence of previous myocardial infarction (more than three months before), and one presented three months after coronary artery bypass grafting for three vessel disease. Two patients had
Dr Paul.

Accepted for publication 29 January 1999 
Table 1 Patient profile

\begin{tabular}{|c|c|c|c|c|c|c|c|c|}
\hline \multirow[b]{2}{*}{ No } & \multirow[b]{2}{*}{ Age/sex } & \multirow[b]{2}{*}{ Clinical details } & \multicolumn{2}{|c|}{ Cardiac catheterisation } & \multirow[b]{2}{*}{ Arrhythmia } & \multirow{2}{*}{$\begin{array}{l}\text { Period of } \\
\text { IABP support }\end{array}$} & \multirow[b]{2}{*}{ Outcome } & \multirow{2}{*}{$\begin{array}{l}\text { Survival at last } \\
\text { review (months) }\end{array}$} \\
\hline & & & $E F \%$ & Coronary angiogram & & & & \\
\hline 1 & $47 \mathrm{M}$ & $\begin{array}{l}3 \text { days post-MI; } \\
2 \text { vessel angioplasty }\end{array}$ & 40 & 2 vessel & $\mathrm{VT} / \mathrm{VF}$ & 3 days & Med & 11 \\
\hline 2 & $57 \mathrm{M}$ & 10 days post-MI & 40 & 3 vessel & IMVT & 3 days & Died $^{\star}$ & \\
\hline 3 & $63 \mathrm{M}$ & 10 days post-MI & 40 & 1 vessel & IMVT & 4 days & Med & 14 \\
\hline 4 & $64 \mathrm{~F}$ & 15 days post-MI & 40 & 2 vessel & $\mathrm{VT} / \mathrm{VF}$ & 5 days & Med & 2 \\
\hline 5 & $54 \mathrm{M}$ & 15 days post-MI & 35 & 3 vessel & VT & 4 days & OCTx & 12 \\
\hline 6 & $69 \mathrm{M}$ & $\begin{array}{l}3 \text { months } \\
\text { post-CABG }\end{array}$ & 50 & $\begin{array}{l}3 \text { vessel; } 4 / 4 \text { patent } \\
\text { grafts }\end{array}$ & IMVT & 2 days & Med & 4 \\
\hline 7 & $52 \mathrm{M}$ & Old MI; acute LVF & 10 & 3 vessel & VT & 4 days & HCTx & 48 \\
\hline 8 & $54 \mathrm{M}$ & Old MI; acute LVF & 25 & 3 vessel & $\mathrm{VT} / \mathrm{VF}$ & 4 days & Med & 51 \\
\hline 9 & $47 \mathrm{M}$ & Old MI; acute LVF & 20 & 2 vessel & $\mathrm{VT} / \mathrm{VF}$ & 10 days & OCTx & 42 \\
\hline 10 & $55 \mathrm{M}$ & Old MI; acute LVF & 25 & 2 vessel & IMVT & 7 days & $\begin{array}{l}\text { LVAD } \\
\text { inserted }\end{array}$ & 159 \\
\hline 11 & $63 \mathrm{M}$ & Old MI & 30 & 1 vessel & IMVT & 4 days & Med & 42 \\
\hline 12 & $44 \mathrm{M}$ & Old MI & 10 & 3 vessel & IMVT & 10 days & OCTx & 26 \\
\hline 13 & $67 \mathrm{~F}$ & Old MI & 30 & 1 vessel & IMVT & 8 days & Med & 54 \\
\hline 14 & $46 \mathrm{M}$ & Old MI & 20 & 2 vessel & VT & 9 days & Med & $60 \ddagger$ \\
\hline 15 & $63 \mathrm{M}$ & Old MI & 20 & 3 vessel & IMVT & 3 days & Med/AICD & 26 \\
\hline 16 & $59 \mathrm{M}$ & Old MI & 20 & 1 vessel & $\mathrm{VT} / \mathrm{VF}$ & 4 days & OCTx & Died $\$$ \\
\hline 17 & $67 \mathrm{M}$ & Old MI & 20 & 2 vessel & IMVT & 1 day & $\begin{array}{l}\text { Endocard } \\
\text { resection }\end{array}$ & 7 \\
\hline 18 & $72 \mathrm{M}$ & Old MI & 20 & 3 vessel & $\mathrm{VT} / \mathrm{VF}$ & 3 days & Med & 33 \\
\hline 19 & $62 \mathrm{~F}$ & $\begin{array}{l}\text { Dilated } \\
\text { cardiomyopathy }\end{array}$ & 40 & Normal coronaries & $\mathrm{VT} / \mathrm{VF}$ & 7 days & Died $\dagger$ & \\
\hline 20 & $51 \mathrm{M}$ & Cardiac amyloid & 30 & Normal coronaries & VT & 9 days & Med & 34 \\
\hline 21 & $64 \mathrm{~F}$ & $\begin{array}{l}\text { Dilated } \\
\text { cardiomyopathy }\end{array}$ & 35 & Normal coronaries & IMVT & 3 days & Med/AICD & 1 \\
\hline
\end{tabular}

AICD, automatic implanted cardioverter-defibrillator; F, female; HCTx, heterotopic heart transplantation; IABP, intra-aortic balloon pump; IMVT, incessant monomorphic ventricular tachycardia; LVAD, left ventricular assist device; LVF, left ventricular failure; M, male; MI, myocardial infarct; OCTx, orthotopic heart transplantation; VF, ventricular fibrillation; VT, ventricular tachycardia.

$\star$ Died from arrhythmic event before discharge.

tActive treatment withdrawn; died from cardiac failure.

\$Elective orthotopic heart transplant at 6 months; alive at 60 months.

$\S$ Died at 5 months from acute rejection.

TOrthotopic heart transplant at 7 months; alive at 15 months.

dilated cardiomyopathy, and one was subsequently found to have cardiac amyloidosis.

Of the 21 patients, 10 presented with incessant monomorphic ventricular tachycardia (IMVT) and 11 with paroxysmal ventricular tachycardia and/or ventricular fibrillation (VT/ $\mathrm{VF})$.

The management of the patients before counterpulsation is summarised in table 2 . Given the extended period over which patients were admitted and the varying clinical circumstances there are understandable variations in management.

Table 2 Treatment before intra-aortic balloon counterpulsation

\begin{tabular}{lllllll}
\hline Patient & Arrhythmia & $\begin{array}{l}\text { Antiarrhythmic } \\
\text { drugs }\end{array}$ & CV & Pace & $\begin{array}{l}\text { Continuous } \\
\text { atrial pacing }\end{array}$ & $\begin{array}{l}\text { Catheter } \\
\text { ablation }\end{array}$ \\
\hline 1 & VT/VF & A,L,P,Mg & + & - & - & - \\
2 & IMVT & A,P,Mg,L,F,Br,B & - & + & + & + \\
3 & IMVT & A,L,Mg & + & + & + & - \\
4 & VT/VF & A,L,Br & + & + & + & - \\
5 & VT & A & + & + & + & - \\
6 & IMVT & A,B,M,Mg & + & + & - & - \\
7 & VT & A,L,Mg & + & - & - & - \\
8 & VT/VF & A & + & - & - & - \\
9 & VT/VF & A & + & - & - & - \\
10 & IMVT & A,L,Mg & + & + & + & - \\
11 & IMVT & A,D,L,Mg,E & - & + & - & - \\
12 & IMVT & A,L,Mg & - & + & + & - \\
13 & IMVT & A,L,Mg,E,P & + & + & + & - \\
14 & VT & A,F,L,Mg & + & - & - & - \\
15 & IMVT & A,L,Mg & + & + & - & - \\
16 & VT/VF & A & + & - & - & - \\
17 & IMVT & A,P & + & + & - & - \\
18 & VT/VF & A,P,F & + & + & - & - \\
19 & VT/VF & A,V,L,B & + & + & - & - \\
20 & VT & A,Mg & + & - & - & + \\
21 & IMVT & A,L,Mg & + & + & + & -
\end{tabular}

$\mathrm{A}$, amiodarone; $\mathrm{B}, \beta$ blocker; $\mathrm{Br}$, bretylium; $\mathrm{CV}$, dc cardioversion; $\mathrm{D}$, disopyramide; $\mathrm{E}$, esmolol; $\mathrm{F}$ flecainide; IMVT, incessant monomorphic ventricular tachycardia; L, lignocaine (lidocaine); $\mathrm{M}$, mexilitine; $\mathrm{Mg}$, magnesium; $\mathrm{P}$, procainamide; Pace, termination by pacing; $\mathrm{V}$, verapamil, VF, ventricular fibrillation; VT, ventricular tachycardia.
Before transfer all patients had been loaded with intravenous amiodarone (mean cumulative dose $6.8 \mathrm{~g}$, range 1.2 to $12 \mathrm{~g}$ ), and $17 \mathrm{had}$ received between one and six additional antiarrhythmic agents (median of two drugs). Suppression of the arrhythmia by overdrive ventricular or atrial pacing had been attempted without success in 11 patients.

On transfer the electrolyte status was checked and where necessary abnormalities were rectified. Amiodarone treatment was continued but all other antiarrhythmic agents were discontinued. Magnesium and $\beta$ blockers were given in some patients but no additional antiarrhythmic agents were used. There was clinical evidence of reversible ischaemia in only one patient. In this patient, angiography showed two vessel disease and impaired ventricular function. Coronary angioplasty was performed on both vessels, but without resolution of the ventricular arrhythmia.

Coronary disease was evident in 18 of the 21 patients on angiography and all had impaired left ventricular function. The mean ejection fraction was $29 \%$ (range $10 \%$ to $50 \%$ ) and mean left ventricular end diastolic pressure was measured at $25 \mathrm{~mm} \mathrm{Hg}$ (range 14 to $50 \mathrm{~mm}$ $\mathrm{Hg}$ ). The morbidity associated with emergency surgical revascularisation in haemodynamically unstable patients is well recognised and in the absence of evidence of reversible ischaemia this was not considered.

Ventricular and atrial pacing wires were inserted under fluoroscopic control in 14 patients and attempts to terminate the arrhythmia by pacing were made. Eight of these 
Table 3 Results of treatment with intra-aortic balloon counterpulsation (IABCP)

\begin{tabular}{llll}
\hline & \multicolumn{2}{l}{ Episodes of sustained ventricular tachycardia } \\
\cline { 2 - 4 } Patient & Before IABCP & During IABCP & After IABCP \\
\hline 1 & $>20$ & 4 & 0 \\
2 & $>20$ & 0 & 1 \\
3 & $>20$ & 0 & 0 \\
4 & 18 & 0 & 0 \\
5 & $>20$ & 0 & - \\
6 & $>20$ & 3 & 1 \\
7 & 4 & 0 & - \\
8 & 4 & 0 & 1 \\
9 & 6 & 0 & - \\
10 & $>20$ & 13 & 0 \\
11 & $>20$ & 1 & 3 \\
12 & $>20$ & 0 & - \\
13 & $>20$ & 0 & 0 \\
14 & 7 & 0 & 0 \\
15 & 10 & 8 & 0 \\
16 & 4 & 0 & - \\
17 & $>20$ & 1 & 1 \\
18 & 12 & 0 & 3 \\
19 & 7 & 0 & 0 \\
20 & $>20$ & 0 & 0 \\
21 & $>20$ & $>20$ & 2 \\
\hline
\end{tabular}

patients were continuously paced at accelerated atrial rates, with only partial control of arrhythmia. Catheter ablation was attempted in one patient.

All patients continued to have frequent ventricular arrhythmia despite the measures outlined above. The use of IABCP was primarily to control the ventricular arrhythmia, although six patients had sufficient haemodynamic compromise for inotropic support.

The intra-aortic balloon pump (IABP) was inserted percutaneously under local anaesthesia through the right femoral artery and positioned in the descending thoracic aorta distal to the left subclavian artery. Counterpulsation was immediately initiated. IABCP was timed to inflate and deflate in synchronisation with the mechanical cardiac cycle triggered by the $\mathrm{R}$ wave on the ECG.

At the time of IABP insertion, 11 patients with paroxysmal VT/VF were in sinus or paced rhythm, in two cases after repeated dc cardioversion. Counterpulsation was used with a $2: 1$ ratio during arrhythmia and a $1: 1$ ratio when sinus rhythm was present in the 10 patients with incessant ventricular tachycardia. In these patients sinus rhythm was achieved by pace termination in three patients, dc cardioversion in three, and both methods in four.

\section{Results}

The use of IABPC reduced the frequency of ventricular arrhythmia and stabilised the patient's general condition in 18 of the 21 cases. There were no complications related to the use of the IABP itself. Complete abolition of ventricular arrhythmia was achieved in 14 patients and significant reduction in episodes of sustained ventricular tachycardia in four (table 3). In the 10 patients with incessant monomorphic ventricular tachycardia, IABCP resulted in successful termination of the arrhythmia (using the treatments described above in Methods) within 30 to 85 minutes.

Removal of the IABP was considered after at least 24 hours of haemodynamic and arrhythmic stability. Ventricular arrhythmia was con- trolled to the extent that it was possible to withdraw mechanical support in 13 patients. Stabilisation of ventricular arrhythmia on medical treatment was maintained in 10 of these patients. Of the remaining three patients, one underwent endocardial resection. Another patient had evidence of progressive heart failure caused by cardiac amyloidosis with associated systemic involvement. This patient was not considered suitable for cardiac transplantation and active treatment was withdrawn. The third patient suffered a fatal in-hospital arrest five days after removal of the balloon.

Control of the arrhythmia was achieved in five patients, but because of severe ventricular impairment or dependence on mechanical support, cardiac transplantation was considered the optimal definitive management and these were continued on IABCP until they successfully underwent cardiac transplantation.

In total, 12 patients were eventually stabilised on medical treatment. Following stabilisation all patients underwent programmed ventricular stimulation. Ventricular tachycardia remained inducible in five patients, but in two the arrhythmia was haemodynamically unstable and these patients underwent implantation of a cardioverter-defibrillator.

IABCP failed to produce any improvement in the clinical situation in three patients and was subsequently discontinued. Amiodarone alone was effective in reducing the frequency of ventricular arrhythmia in two of these patients; however, haemodynamically unstable arrhythmia was still inducible during programmed stimulation and these patients therefore underwent implantation of a transvenous defibrillator. In the third patient, further haemodynamic deterioration necessitated the use of a left ventricular assist device before transplantation. IABCP was continued for a mean period of 5.1 days (range 1 to 10 days).

Nineteen patients were discharged from hospital and were followed as outpatients. During a mean follow up period of 25.7 months the overall survival was $95 \%$. There have been no late deaths from cardiac arrhythmia.

\section{Discussion}

Our experience shows that, in selected patients, the use of intra-aortic balloon counterpulsation can contribute to the management of medically refractory ventricular arrhythmia. That there were no complications related to the percutaneous insertion of the balloon catheter shows that this treatment can be used with a relatively high degree of safety in such patients. Our experience is supported by a larger survey that describes a complication rate of $4.6 \%$ in a series of 231 patients. $^{4}$

We believe that the use of mechanical support is of potential benefit in two groups of patients. The first group is those with severely impaired ventricular function in whom the development of ventricular arrhythmia suggests end stage cardiac failure. These patients tolerate negative inotropic agents poorly and will ultimately require cardiac transplantation as definitive treatment. As a 
supraregional transplant centre we have been able to provide such treatment, which undoubtedly contributes to the overall success of this approach.

The second group is those in whom either the arrhythmia itself or the administration of antiarrhythmic drugs has compromised a normally stable haemodynamic state. It is of interest that although the haemodynamic support provided was only temporary, it was sufficient to provide a therapeutic window for the stabilisation and subsequent discharge of patients on medical treatment alone (usually amiodarone). It is possible, therefore, that use of IABCP simply allowed time for effective antiarrhythmic treatment to work, or to allow washout of proarrhythmic drugs.

Patients with incessant ventricular tachycardia are usually considered candidates for radiofrequency ablation, although this does have associated risk. In the 10 patients with IMVT in this series, eight had achieved control with IABCP, remaining stable after IABCP cessation on medical treatment and thus avoiding the need for radiofrequency ablation.

The mechanism by which IABCP helps control ventricular arrhythmia is unclear, but we believe that there are several possible effects, both direct and indirect. IABCP has been shown to increase peak aortic diastolic pressure and to decrease peak left ventricular systolic pressure. ${ }^{5-8}$ This augmentation of the coronary perfusion pressure enhances coronary blood flow and therefore myocardial oxygen supply. ${ }^{9-14}$ Furthermore systolic left ventricular offloading reduces myocardial wall tension and oxygen demand. ${ }^{6-81516}$ Although these effects will reduce the potential for ischaemia to contribute to arrhythmogenesis, it is probable that this mechanism is of minor importance. The patients with normal coronary arteries still benefited from IABCP, and reversible ischaemia was a clinically demonstrable problem in only one patient with ischaemic heart disease.

A further indirect effect of IABCP on arrhythmogenesis may be through a reduction in the adrenergic drive as a result of supporting the haemodynamic state and the associated use of sedation. High adrenergic levels are known to be proarrhythmic and reduction to be protective.

Of greater interest is the possibility that the mechanical effects of the IABCP in themselves had a direct effect in controlling the arrhythmia. The concept of mechano-electrical feedback has been derived from numerous studies showing the influence of alterations in preload and afterload on arrhythmogenesis in both isolated tissue and animal hearts. ${ }^{17-20}$ Experiments in anaesthetised dogs have shown that progressive increase in afterload may result in ventricular ectopy and tachycardia, ${ }^{18}$ and that the threshold for the development of these arrhythmias is reduced in the presence of induced coronary artery disease. Stretching of normal cardiac tissue (increasing preload) results in a reduction of action potential duration and spontaneous depolarisation. ${ }^{21-24}$ Taggart et al reported similar changes in action potential duration in patients coming off cardiopulmonary bypass. ${ }^{25}$ Clinically, the association of heart failure with ventricular arrhythmias and improvement in prognosis with certain vasodilators is well established. ${ }^{26}$ It is believed that mechanical events may play at least a part in this process.

\section{STUDY LIMITATIONS}

This was a retrospective review of our clinical practice and consequently detailed haemodynamic and electrophysiological data are not complete. Prospective analysis would be required to provide this information. As a result, it is difficult to explain why the arrhythmia remained suppressed after cessation of IABCP in some patients while it was ineffective at any stage in others. Furthermore, it is not possible to make any definitive comments about the likely mechanism of action. It is clear, however, that in a significant number of patients this form of treatment is of use in aiding control of malignant ventricular arrhythmia when conventional treatment has failed or is slow to be effective. We believe therefore that IABCP should be considered in patients with refractory ventricular arrhythmia and impaired ventricular function.

1 Willerson JT, Curry GC, Watson JT, et al. Intra-aortic balloon counterpulsation in patients in cardiogenic shock, medically refractory left ventricular failure and/or recurrent ventricular tachycardia. Am $\mathcal{f}$ Med 1975;58:183-91.

2 Hanson EC, Levine FH, Kay HR, et al. Control of post-infarction ventricular irritability with the intra-aortic post-infarction ventricular irritability with

3 Culliford AY, Madden MR, Isom OW, et al. Intra-aortic balloon counterpulsation: refractory ventricular tachycardia. loon counterpulsation:

4 Eltchaninoff H, Dimas AP, Whitlow PL. Complications associated with percutaneous placement and use of intra-aortic balloon counterpulsation. Am f Cardiol 1993; 71:328-32.

5 Maccioli GA, Lucas WJ, Norfleet EA. The intra-aortic balloon pump: a review. F Cardiothorac Anesth 1988;2:365-73.

6 Urschel CW, Eber L, Forester J, et al. Alteration of mechanical performance of the ventricle by intra-aortic balloon counterpulsation. Am 7 Cardiol 1970;25:546-51.

7 Mullins CB, Sugg WL, Kennelly BM, et al. Effect of arterial counterpulsation on left ventricular volume and pressure. Am F Physiol 1971;220:694-8.

8 Powell WJ, Daggett WM, Magro AE, et al. Effects of intraaortic balloon counterpulsation on cardiac performance, oxygen consumption and coronary blood flow in dogs. Circ Res 1970;26:753-64.

9 Fuchs RM, Brin KP, Brinker JA, et al. Augmentation of regional coronary blood flow by intra-aortic balloon counterpulsation in patients with unstable angina. Circulation 1983;68:11-23

10 Saini VK, Hood WB, Hechtman HB, et al. Nutrient myocardial blood flow in experimental myocardial ischaemia: effects of intra-aortic balloon counterpulsation and coronary reperfusion. Circulation 1975;52:1086-90.

11 Swank M, Singh HM, Flemma RJ, et al. Effect of intra-aortic balloon pumping on nutrient coronary flow in normal and ischaemic myocardium. I Thorac Cardiovasc Surg 1978;76:538-44.

12 Kern MJ, Aguirre FV, Tatineni S, et al. Enhanced coronary blood flow velocity during intra-aortic balloon counterpulsation in critically ill patients. F $\mathrm{Am}$ Coll Cardiol 1993;21:359-68.

13 Kern MJ, Aguirre F, Bach R, et al. Augmentation of coronary blood flow by intra-aortic balloon pumping in patients ary blood flow by intra-aortic balloon pumping in patie

14 Bregman D, Parodi EN, Edie RN, et al. Intra-operative unidirectional intra-aortic balloon pumping in the management of left ventricular power failure. $\mathcal{F}$ Thorac Cardiovasc Surg 1975;70:1010-23.

5 Gerweirtz H, Ohley W, Williams DO, et al. Effect of intra-aortic balloon counterpulsation on regional myocardial blood flow and oxygen consumption in the presence of coronary artery stenosis: observation in an awake animal model. Am f Cardiol 1982;50:829-37.

16 Williams DO, Korr KS, Gewirtz H, et al. The effect of intraaortic balloon counterpulsation on regional myocardial blood flow and oxygen consumption in the presence of coronary artery stenosis in patients with unstable angina. Cironary artery stenosis in
culation 1982;66:593-7.

17 Sideris DA, Rokas SG, Kontoyannis DA. Pressure related ventricular tachycardia. Int $\mathcal{F}$ Cardiol 1990;28:382-3.

18 Hansen DE, Grig CS, Hondenghem LM. Stretch-induced arrhythmias in the isolated canine ventricle. Evidence for 
the importance of mechano-electrical feedback. Circulation 1990;81:1094-105.

19 Reiter MJ, Synhorst DP, Mann DE. Electrophysiological effects of acute ventricular dilatation in the isolated rabbit heart. Circ Res 1988;62:554-62.

20 Franz R, Burkhoff D, Yue DT, et al. Mechanically induced action potential changes and arrhythmia in isolated and in situ canine hearts. Cardiovasc Res 1989;23:213-23.

21 Taggart P, Sutton P, John R, et al. Monophasic action potential recordings during acute changes in ventricular loading induced by the Valsalva manoeuvre. Br Heart $f$ 1992;67:221-19.

22 Dean JW, Lab MJ. Effects of changes in load on monophasic action potential and segment length of pig heart in situ. Cardiovasc Res 1989;43:887-96.
23 Coulshed DS, Hainsworth R, Cowan JC. The influence of myocardial systolic shortening on action potential duration following changes in left ventricular end-diastolic pressure.

24 Franz MR, Cima R, Wang D, et al. Electrophysiological effects of myocardial stretch and mechanical determinants of stretch-activated arrhythmias. Circulation 1992;86:96878

25 Taggart P, Sutton PM, Treasure T, et al. Monophasic action potentials at discontinuation of cardiopulmonary bypass: evidence for contraction-excitation feedback in man. Circulation 1988;77:1266-1275.

26 Dean JW, Lab MJ. Arrhythmia in heart failure; role of mechanically induced changes in electrophysiology. Lancet 1989;i:1302-12.

\section{Candida endocarditis of the right heart}

An 84 year old woman was referred for surgical evaluation. Her clinical history was characterised by hypertension and a permanent pacemaker implanted in

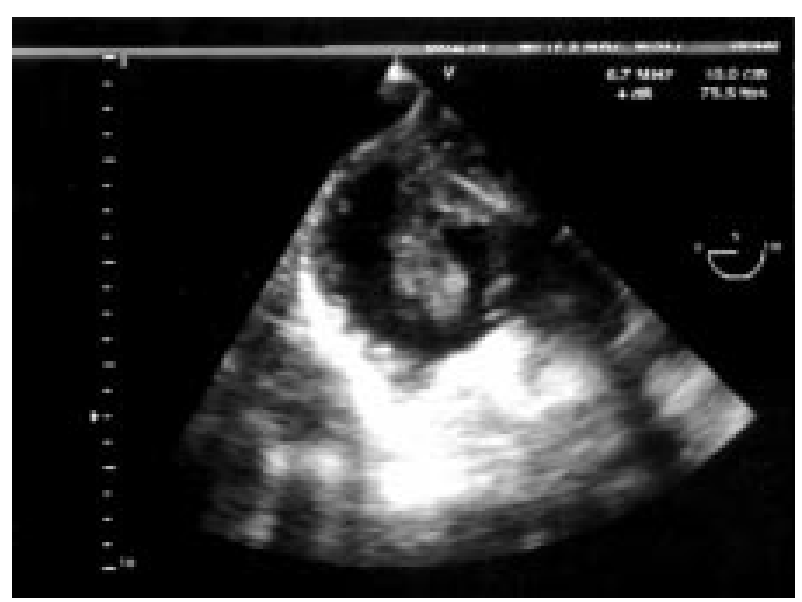

June 1997 because of complete atrioventricular block. Two months before, she had undergone successful antibiotic treatment for a gastrointestinal infection; one month after discharge she had septic fever and a new diastolic murmur. Blood cultures were positive for Candida albicans.

Transthoracic echocardiography showed normal cardiac sections and a large mass in the right atrium prolapsing into the tricuspid valve orifice. Transoesophageal echocardiography (pictured) showed two large vegetations: one attached to the pacemaker catheter; the second free in the right atrium and connected to the first vegetation. Both vegetations prolapsed into the tricuspid valve during diastole. There were no signs of pulmonary involvement. The patient did not undergo surgery because her general clinical condition was very compromised and the surgical risk too high.

MARIA CRISTINA BARATELLA MAURIZIO DAN ALESSANDRO FABBRI 